\title{
TORSIONFREE PROJECTIVE MODULES
}

\author{
MARK L. TEPLY
}

ABSTRACT. In this paper, the following condition for a torsion theory in the sense of S. E. Dickson is examined:

(P) Every nonzero torsionfree module contains a nonzero projective submodule.

A special relationship between condition $(\mathrm{P})$ and Goldie's torsion theory is shown; and the rings, for which every nontrivial torsion theory satisfies condition $(\mathrm{P})$, are classified.

1. Introduction. It is well known that in the category of unitary modules over a commutative integral domain, every torsionfree cyclic module is projective; and every finitely generated torsionfree module is projective if and only if $R$ is a Prüfer domain. Recent papers ([8] and [10]) consider similar properties for Dickson's torsionfree modules [5] over more general rings. The connections between projective and torsionfree modules are closely related to splitting problems, which are currently being studied (e.g. see [3], [9], [10]). The projectivity condition $(\mathrm{P})$, which is studied in this paper, is more general than the ones studied in [8] and [10].

2. Preliminaries. In this paper all rings $R$ have an identity element and all modules are unitary left $R$-modules. ${ }_{R} \Re$ denotes the category of left $R$-modules.

As in [5], a nonempty subclass $J$ of ${ }_{R} \mathfrak{T}$ is called a torsion class if $J$ is closed under factors, extensions, and arbitrary direct sums. For each module $A$, a torsion class $\mathfrak{I}$ determines a unique maximal $\mathfrak{J}$ submodule, $\Im(A)$, and $\Im(A / J(A))=0$. The class

$$
\mathcal{F}=\left\{A \in \in_{R} \mathfrak{T} \mid \mathfrak{T}(A)=0\right\}
$$

is called the torsionfree class corresponding to $\mathfrak{J}$, and the pair $(\mathfrak{J}, \mathcal{F})$ is called a torsion theory for ${ }_{n} \mathfrak{T}$. Modules in $J$ are called torsion; modules in $\mathcal{F}$ are called torsionfree. A torsion theory $(\mathcal{F}, \mathcal{F})$ is called trivial if either $J=\{0\}$ or $\mathcal{F}=\{0\}$; any other torsion theory is nontrivial. If $J$ is closed under submodules, then $(J, F)$ is called hereditary. In this case $J$ is precisely a class of negligible modules associated with a topologizing and idempotent filter of left ideals in the sense of P. Gabriel [6]. Such a filter will be denoted by $F(\mathfrak{J})$.

Received by the editors April 20, 1970.

AMS 1970 subject classifications. Primary 16A50, 18E40; Secondary 16A48.

Key words and phrases. Ring, projective module, torsion theory, torsion, torsionfree, essential submodule, perfect ring, socle. 
3. Goldie's torsion theory and condition $(\mathrm{P})$. Condition $(\mathrm{P})$ turns out to be strongly related to Goldie's torsion theory $(\mathcal{G}, \Re)$. Goldie's torsion class $G$ for ${ }_{R} \mathfrak{T}$ is precisely the class of modules with essential singular submodule; while Goldie's torsionfree class $\Re$ is the class of nonsingular modules. The filter $F(\mathcal{G})$ is the smallest topologizing and idempotent filter containing all the essential left ideals of $R$. Papers [1] and [8] contain further properties of $(\mathcal{G}, \Re)$.

We begin our results by formulating a tool for studying condition (P).

Proposition 1. If the torsion theory $(\mathcal{J}, \mathcal{F})$ satisfies condition (P),

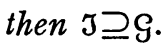

Proof. Since the Goldie torsion class $g$ is the smallest torsion class containing every singular cyclic module (see [1]), it is sufficient to show that $R / I \in J$ for every essential left ideal $I$ of $R$. So let $I$ be an essential left ideal of $R$, and let $J(R / I)=K / I \neq R / I$. By condition (P), $R / K$ contains a nonzero projective submodule $B / K$, and thus $B=K \oplus L$, where $L \cong B / K$. Therefore $L \cap I \subseteq L \cap K=0$, contradicting the fact that $I$ is essential in $R$. Hence $J(R / I)=R / I$ as desired.

In [6], P. Gabriel has called a torsion class $J$ stable if $E(T) \in J$ for all $T \in J$.

Now suppose that $(\Im, \mathcal{F})$ is a torsion theory such that $\Im \supseteq g$. Since $E(T) / T \in \mathcal{G}$ for all $T \in \mathcal{J}$, it follows from $J$ closed under extensions that $E(T) \in J$. Consequently, $\mathfrak{J}$ is a stable class, and we have the following corollary to Proposition 1:

COROLLARY. If the torsion theory $(\mathcal{J}, \mathcal{F})$ satisfies condition $(\mathrm{P})$, then I is stable.

Next we point out some conditions equivalent to condition (P).

Proposition 2. The following statements are equivalent for a torsion theory $(\mathcal{J}, \mathcal{F})$ :

(1) $(\mathfrak{J}, \mathcal{F})$ satisfies condition (P).

(2) Every nonzero left ideal in $\mathcal{F}$ contains a nonzero projective left ideal, and $\Im \supseteq g$.

(3) If $0 \neq R / I \in \mathcal{F}$, then there exists a nonzero projective left ideal $K$ such that $K \cap I=0$.

Proof. (1) $\Rightarrow(2)$ follows from Proposition 1, and (3) $\Rightarrow(1)$ is clear.

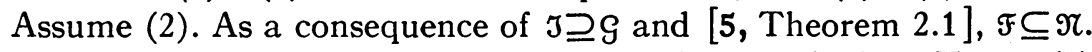
So if $R / I \in \mathcal{F}$, then $I$ is not an essential left ideal of $R$. Hence (3) follows from the first part of (2). 
If the torsion theory $(\mathfrak{J}, \mathfrak{F})$ satisfies condition $(\mathrm{P})$ and if $\left(\mathcal{J}^{\prime}, \mathfrak{F}^{\prime}\right)$ is any torsion theory with $J^{\prime} \supseteq \mathcal{F}$, then $\mathcal{F}^{\prime} \subseteq J$. Hence $\left(J^{\prime}, \mathcal{F}^{\prime}\right)$ also satisfies condition $(\mathrm{P})$. So whenever $(\mathcal{G}, \Re)$ satisfies condition $(\mathrm{P})$, we obtain information about all torsion theories $\left(J^{\prime}, \mathcal{F}^{\prime}\right)$ such that $J^{\prime} \supseteq \mathcal{G}$. Moreover, Proposition 1 shows $G$ is the smallest torsion class for ${ }_{R} \mathbb{M}$ that can satisfy condition (P). Applying Proposition 2, the reader can verify that $(\mathcal{G}, \mathfrak{r})$ satisfies condition $(P)$ for any of the following classes of rings:

(a) rings [8] for which nonsingular principal left ideals are projective,

(b) rings $R$ for which the left socle of $R$ is essential in $R$,

(c) self-injective rings, and

(d) rings with essential left singular ideal.

The rings in class (a) include rings without zero divisors, von Neumann regular rings, and left semihereditary rings; the rings in class (b) include the right perfect rings and left Artinian rings.

4. The main results. Next we turn our attention to classifying the rings whose nontrivial torsion theories all satisfy condition $(\mathrm{P})$. This turns out to be primarily a study of certain rings with only one simple module (up to isomorphism). Consequently, we need a torsion class dealing with simple modules, namely Dickson's simple torsion class $\mathcal{S}[5]$. $\mathcal{S}$ consists of the modules $M$ such that every nonzero homomorphic image of $M$ has nonzero socle.

Before stating the theorem, we give a lemma that we shall need.

Lemma 1. Let $R$ be a ring with only one simple module (up to isomorphism) and Jacobson radical $N$. Then either $N=0$ or $N$ is an essential left ideal.

Proof. Since $R$ has a unique simple module, then $N$ is the unique maximal two-sided ideal of $R$. If $I$ is a nonzero left ideal and $I \cap N=0$, then it follows that $I R=R$ by the unique maximality of $N$. Thus for $x \in N, x \in N=N R=N I R=0$.

THEOREM 1. Every nontrivial torsion theory for ${ }_{R} \mathrm{TT}$ satisfies condition $(\mathrm{P})$ if and only if $R$ is one of the following types of rings:

(1) semisimple Artinian rings;

(2) rings which are left and right perfect and which have a unique maximal two-sided ideal;

(3) simple rings with the following properties:

(a) Nonzero modules have maximal submodules.

(b) If $I$ is a proper essential left ideal, then $\operatorname{Soc}(R / I) \neq 0$. 
(c) Every nonzero left ideal contains a nonzero projective left ideal.

(d) Any two simple modules are isomorphic.

REMARKs. Examples of type (3) rings, which are not division rings, can be found in [4], where $V$-rings are studied. A $V$-ring is a ring for which every simple module is injective. It may be an interesting question to determine which rings of type (3) are $V$-rings. The rings of types (1) and (2) are the extreme cases, i.e. $\mathcal{G}=0$ or $\mathcal{G}={ }_{R} \mathfrak{T}$.

Proof. "Only if": First, suppose that $R$ has at least two nonisomorphic simple modules, $U$ and $V$. We form $\left(J_{U}, \Im_{U}\right)$ and $\left(\Im_{V}, \mathcal{F}_{V}\right)$, where $J_{U}$ and $J_{V}$ are the smallest torsion classes containing $U$ and $V$, respectively. Since $U \in J_{U}, V \in \mathcal{F}_{U}, V \in J_{V}$, and all simple modules not isomorphic to $V$ are in $\mathcal{F}_{V}$, then these are nontrivial torsion theories. So applying the hypothesis to $\left(J_{U}, \mathscr{F}_{U}\right)$ and $\left(J_{V}, F_{V}\right)$, we see that every simple module is projective. But this is well known to imply that $R$ is a semisimple Artinian ring.

For the remainder of the "only if" part of the proof, we may assume that there exists only one simple module $S$ (up to isomorphism), and $S$ is not projective. But then the torsion class

$$
J=\left\{M \in_{R} \mathfrak{T l} \mid \operatorname{Hom}(M, S)=0\right\}
$$

must be trivial. (Otherwise, $S$ is projective.) Thus $J=0$, and every nonzero module contains a maximal submodule. Hence every nonzero torsion class (being closed under homomorphic images) contains $S$. In particular, $S \in \mathcal{G}$. But $\delta$ is the smallest torsion class containing $S$; so $\delta \subseteq \mathcal{G}$. Since $\delta \neq\{0\}$, then $\delta=\mathcal{G}$ by Proposition 1 . So if $I$ is a proper essential left ideal, then $\operatorname{Soc}(R / I) \neq 0$.

Let $N$ be the Jacobson radical of $R$. It follows from Lemma 1 that either $N=0$ or $N$ is an essential left ideal; moreover, $N$ is the unique maximal two-sided ideal of $R$. In case $N=0, R$ must be a simple nonsingular ring with a cofinal subset of nonzero projective left ideals. Combining this with the preceding paragraph, we see that $R$ is of type (3) whenever $N=0$ and the unique simple module of $R$ is not projective.

Thus for the remainder of the proof of the "only if" part, we also assume that $N$ is an essential left ideal. Since $\mathcal{G}=S$, $\operatorname{Soc}(R / N) \neq 0$. But $N$ is the unique maximal two-sided ideal of $R$; so it follows that $R / N$ is a simple Artinian ring. Since $N$ is left $T$-nilpotent by [7, Lemma 1], it follows from [2, Theorem 6.3], that

$$
0=1 \mathrm{FPD}(R)=\sup \{\operatorname{hd}(M) \mid \operatorname{hd}(M)<\infty\} .
$$

Let $L$ be a left ideal which is maximal with respect to $L \subseteq N$ and 
$L \cap g(R)=0$. By condition $(\mathrm{P})$, there exists an essential projective submodule $A$ of $L$. Since $N$ is essential in $R$, then $A \oplus g(R)$ is also an essential left ideal of $R$. Now the exact sequence

$$
0 \rightarrow A \rightarrow R \rightarrow R / A \rightarrow 0
$$

yields $h d(R / A) \leqq 1$. But $1 \mathrm{FPD}(R)=0$; hence $\operatorname{hd}(R / A)=0$. Thus $R=A \oplus G$, where $G \cong R / A$. Since $A \subseteq N$ and $N$ contains no nonzero idempotent elements, it follows that $A=0$. By our construction of $A$, $\mathcal{G}(R)$ is essential in $G=R$. Since $S=\mathcal{G}$ is stable, then $S(R)=R$; whence nonzero modules have nonzero socles. So by $[2$, Theorem $\mathrm{P}], R$ is right perfect, and thus $R$ is of type (2).

"If": If $R$ is a semisimple Artinian ring, the result is clear. The reader can easily verify the result for rings of types (2) and (3) by using the following fact: If $R$ is a left perfect ring, then nonzero modules have maximal submodules [2]; and rings satisfying this latter condition have the property that every nonzero torsion class contains a simple module.

Lemma 2. Suppose that every left ideal of $R$ is an intersection of a summand of $R$ and a left ideal in $F(S)$. Then $S$ is stable; and $\operatorname{Soc}(R / I)$ $=0$ if and only if $I$ is a summand of $R$ containing $\operatorname{Soc}(R)$.

Proof. From the hypothesis it follows that every essential left ideal is in $F(\mathcal{S})$; whence $F(\mathcal{S}) \subseteq F(\mathcal{S})$. Consequently $\mathcal{G} \subseteq \mathcal{S}$, and thus $\delta$ is stable by the remarks preceding the corollary to Proposition 1.

Suppose $\operatorname{Soc}(R / I)=0$, and let $I=L \cap K$, where $L \in F(\delta)$ and $K$ is a summand of $R$. If $\Im$ denotes the torsionfree class for $S$, then

$$
\frac{K+L}{L} \cong \frac{K}{K \cap L}=\frac{K}{I} \in \delta \cap \mathcal{F}=0 .
$$

Hence $K=I$ is a summand of $R$, which clearly contains $\operatorname{Soc}(R)$.

The converse part of the lemma is clear.

Let $S$ be a simple module. As in [5] we let $J_{S}$ denote the smallest torsion class containing $S$. The resulting torsion theory $\left(\mathfrak{J}_{S}, \mathfrak{F}_{S}\right)$ is hereditary.

Next we consider a specialization of Theorem 1.

THEOREM 2. The following statements are equivalent for a ring $R$ :

(1) Every nontrivial hereditary torsion theory $(\mathcal{J}, \mathcal{F})$ has the property: Every cyclic module in $\mathcal{F}$ is projective.

(2) Either (a) $R$ is a semisimple Artinian ring, or else (b) $R$ has a unique simple module $S$ (up to isomorphism) and every left ideal of $R$ is an intersection of a summand of $R$ and a member of $F\left(J_{S}\right)$. 
Proof. (1) $\Rightarrow(2)$. If $R$ possesses two nonisomorphic simple modules, then the first part of the proof of Theorem 1 shows that $R$ must be a semisimple Artinian ring. Thus we assume $R$ has only one simple module $S$ (up to isomorphism), and $S$ is not projective.

If $J_{S}={ }_{R} \Re$, then (2) is trivial; so we assume that $J_{S} \neq{ }_{R} \Re$. Let $I$ be a left ideal such that $\Im_{S}(R / I)=K / I \neq R / I$. By (1), $R=K \oplus P$, where $P \cong R / K$ is projective. Then $L=I \oplus P \in F\left(J_{S}\right)$, and $I=K \cap L$ as desired.

$(2) \Rightarrow(1)$. If $R$ is a semisimple Artinian ring, then every module is projective.

If $R$ has a unique simple module, then every nontrivial hereditary torsion theory $(\mathfrak{J}, \mathcal{F})$ satisfies $\mathfrak{J} \supseteq J_{S}=\mathcal{S}$. Hence $\mathfrak{F} \subseteq \mathcal{F}_{S}$. So it is sufficient to show that every cyclic module in $\mathfrak{F}_{S}$ is projective. But if $R / I \in \mathcal{F}_{S}$, then an immediate consequence of (2) and Lemma 2 is that $I$ is a summand of $R$; whence $R / I$ is projective.

\section{REFERENCES}

1. J. S. Alin and S. E. Dickson, Goldie's torsion theory and its derived functor, Pacific J. Math. 24 (1968), 195-203. MR 37 \#2834.

2. H. Bass, Finitistic dimension and a homological generalization of semi-primary rings, Trans. Amer. Math. Soc. 95 (1960), 466-488. MR 28 \#1212.

3. R. L. Bernhardt, Splitting hereditary torsion theories over semi-perfect rings, Proc. Amer. Math. Soc. 22 (1969), 681-687.

4. J. H. Cozzens, Homological properties of the ring of differentiable polynomials, Bull. Amer. Math. Soc. 76 (1970), 75-79.

5. S. E. Dickson, $A$ torsion theory for abelian categories, Trans. Amer. Math. Soc. 121 (1966), 223-235. MR 33 \#162.

6. P. Gabriel, Des catégories abéliennes, Bull. Soc. Math. France 90 (1962), 323448. MR 38 \#1144.

7. R. M. Hamsher, Commutative rings over which every module has a maximal submodule, Proc. Amer. Math. Soc. 18 (1967), 1133-1137. MR 36 \#154.

8. M. L. Teply, Some aspects of Goldie's torsion theory, Pacific J. Math. 29 (1969), 447-459.

9. - Homological dimension and splitting torsion theories, Pacific J. Math. 32 (1970), 193-206.

10. D. Turnidge, Torsion theories and semihereditary rings, Proc. Amer. Math. Soc. 24 (1970), 137-143.

University of Florida, Gainseville, Florida 32601 